\title{
THE SET OF ALL GENERALIZED LIMITS OF BOUNDED SEQUENCES
}

\author{
MEYER JERISON
}

1. Introduction. Let $M$ be the normed linear space whose general element, $x$, is a bounded sequence

$$
\left\{\xi_{n}\right\}_{n=1}^{\infty}
$$

of real numbers, and $\|x\|=1 . u . b .\left|\xi_{n}\right|$. Let $T$ denote the linear operation (of norm 1) defined by $T x=\left(\xi_{2}, \xi_{3}, \ldots, \xi_{n+1}, \ldots\right)$. A generalized limit is a linear functional $\phi$ on $M$ which satisfies the conditions

(1) $x \geqslant 0$ (i.e., $\xi_{n} \geqslant 0$ for all $n$ ) implies $\phi(x) \geqslant 0$;

(2) $\phi(T x)=\phi(x) \quad$ for all $x \in M$;

(3) $\phi(1,1,1, \ldots)=1$.

The set of all generalized limits will be denoted by $L$. In the presence of (1), condition (3) is equivalent to $\|\phi\|=1$.

The basic question of existence of generalized limits has been settled in a variety of ways; the standard proof appears in (2, p. 34). This proof, based upon the Hahn-Banach theorem, actually leads to all generalized limits, and this fact was used in (7) to obtain properties of $L$. In the present paper, attention is focused on another existence proof $(5$, p. $1010 ; \mathbf{8} ; \mathbf{1 0}$, p. 52) which depends, ultimately, on Tychonoff's theorem. In order to describe this proof, we must summarize some well-known properties of $M$ and of the conjugate space $M^{*}(\mathbf{1} ; \mathbf{5})$.

Only one topology in $M^{*}$ will be of interest to us, namely, the weak* topology, which is defined as follows: A directed system $\left\{\phi_{\nu}\right\}$ in $M^{*}$ converges to $\phi$ if $\phi_{\nu}(x) \rightarrow \phi(x)$ for each $x \in M$. An essential property of this topology is that the set

$$
B^{*}=\{\phi|| \phi(x) \mid \leqslant\|x\| \text { for all } x \in M\}
$$

(the unit ball) is compact. $B^{*}$ is also convex, and we are able to apply the Krein-Milman theorem to its subsets. Thus, if $K$ is a closed convex subset of $B^{*}$, and $S(\subset K)$ contains all extreme points of $K$, then $K$ is the closed convex hull of $S$, denoted by $\mathfrak{S}(S)$. In particular, if $K$ is not empty, neither is $S$.

We will denote by $\Omega^{\prime}$ the set of extreme points of $B^{*}$ that satisfy condition (1), or equivalently, the collection of extreme points of the subset of $B^{*}$ which is determined by conditions (1) and (3). Since the latter set is closed and convex, it is, in fact, $\mathfrak{S}\left(\Omega^{\prime}\right)$. Among the functionals in $\Omega^{\prime}$ are those of the form $\theta_{p}(x)=\xi_{p}$ for each fixed natural number $p$. The collection $N$ of all such

Received October 26, 1953; in revised form October 21, 1955. This work was supported, in part, by a grant from the National Science Foundation (USA). 
functionals is a discrete, open, and dense subset of $\Omega^{\prime}$. Since $\Omega^{\prime}$ happens to by compact (1, p. 504), it is the closure of $N$ in $M^{*}$, and it is known as the Stone-Čech compactification of $N$. The set $\Omega$, the complement of $N$ in $\Omega^{\prime}$, is characterized, as a subset of $\Omega^{\prime}$, by the fact that each of its elements satisfies the condition

$\phi(x)$ is independent of the value of $\xi_{n}$ for each fixed $n$.

Its closed convex hull, $\mathfrak{S}(\Omega)$, is the set of all functionals in $M^{*}$ satisfying conditions (1), (3), and (4). Since (2) obviously implies (4), $L \subset \mathfrak{S}(\Omega)$. It is important to note that $L$ is itself a closed convex set.

The proof of existence of generalized limits that was referred to in the second paragraph goes as follows: Let $\chi \in \Omega$, or, more generally, $\chi \in \mathfrak{S}(\Omega)$; a generalized limit, $\psi$, is obtained by setting

$$
\psi(x)=\chi\left(\left\{n^{-1} \sum_{i=1}^{n} \xi_{i}\right\}\right)
$$

for $x=\left\{\xi_{n}\right\}$ in $M$. Now, a functional $\chi \in \mathfrak{S}(\Omega)$ has the property that if $y \in M$ is a convergent sequence, then $\chi(y)$ is the ordinary limit of $y$. If $x$ is a sequence whose arithmetic means, $n^{-1} \sum \xi_{i}$, converge to $\sigma$, say, then $\psi(x)=\sigma$ for every generalized limit $\psi$ which is obtained in this manner. Since it is known that there are some such sequences and some generalized limits which assign to them values different from $\sigma$ (see Theorem 4), it follows that this procedure does not lead to all generalized limits. It is our intention, therefore, to modify this procedure in such a way as to obtain all generalized limits.

Let $T_{\text {" }}$ be the operator

$$
n^{-1} \sum_{i=0}^{n-1} T^{i}
$$

and let $\theta_{1}(x)=\xi_{1}$ for all $x \in M$. Then $\left\{\theta_{1}\left(T_{n} x\right)\right\}$ is the sequence of arithmetic means of $x$, and the generalized limit obtained above may be defined as $\psi(x)=\chi\left(\left\{\theta_{1}\left(T_{n} x\right)\right\}\right)$. From this point of view, an obvious way to generate more generalized limits is to replace $\theta_{1}$ by some other functional. Three observations should be made in this connection.

(a) If $\theta_{1}$ is replaced by $\theta_{p} \in N$, nothing new is obtained, because

$$
\theta_{p}\left(T^{i} x\right)=\theta_{1}\left(T^{i+p-1} x\right), \quad i=1,2, \ldots
$$

so that

$$
\chi\left(\left\{\theta_{p}\left(T_{n} x\right)\right\}\right)=\chi\left(\left\{\theta_{1}\left(T_{n} T^{p-1} x\right)\right\}\right)=\psi\left(T^{p-1} x\right)=\psi(x) .
$$

(b) If $\phi$ is any functional satisfying conditions (1) and (3), i.e., $\phi \in \mathfrak{S}\left(\Omega^{\prime}\right)$, and if $\chi \in \mathfrak{S}(\Omega)$, then

$$
\psi(x)=\chi\left(\left\{\phi\left(T_{n} x\right)\right\}\right)
$$

does define a generalized limit (Theorem 1). 
(c) It is trivial that any generalized limit $\psi$ may be obtained from (5) simply by taking $\phi=\psi$, because $\psi\left(T_{n} x\right)=\psi(x)$ for all $n$.

The purpose of this paper is to prove that the collection $Q$ of all functionals of the form (5) with $\chi$ and $\phi$ in $\Omega$ is sufficient to yield all generalized limits in the sense that $\mathfrak{S}(Q)=L$.

The proof requires a surprising amount of heavy machinery: the representation of $M$ as the space of all continuous functions on $\Omega^{\prime}$, the representation of an arbitrary continuous linear functional on $M$ as a measure on $\Omega^{\prime}$, and one of the deep theorems of measure theory, the individual ergodic theorem. A similar (but apparently weaker) result can be obtained by using entirely different techniques. As was stated before, the set $N$ is dense in $\Omega^{\prime}$, which means that the element $\chi \in \Omega$ is the limit of a directed system

$$
\left\{\theta_{n_{\nu}}\right\}
$$

of elements of $N$. In terms of this directed system, (5) becomes

$$
\psi(x)=\lim _{\nu} \phi\left(T_{n_{\nu}} x\right) .
$$

Since $\chi \in \Omega, n_{\nu} \rightarrow \infty$. Let us consider, now, the set $A$ of all continuous linear functionals of the form

$$
\psi(x)=\lim _{\nu} \phi_{\nu}\left(T_{n_{\nu}} x\right)
$$

where $n_{\nu} \rightarrow \infty, \phi_{\nu} \in \Omega$ for all $\nu$, and the limit is assumed to exist for all $x \in M$. It will be seen that $A$ is a closed subset of $L$, and the proposition $\mathfrak{S}(A)=L$ will be proved independently of the obvious fact that $Q \subset A$. I have not been able to determine ${ }^{1}$ whether $Q$ is a proper subset of $A$ or whether the closure of $Q$ is all of $A$.

2. Limit points of sequences in $M^{*}$. Since it is convenient to work in the space $M^{*}$ as much as possible, we introduce the operator $T^{*}$ on $M^{*}$, defined by $T^{*} \phi(x)=\phi(T x)$ for all $x \in M, \phi \in M^{*}$. Condition (2) becomes $\left(2^{*}\right) T^{*} \phi=\phi$. In keeping with the notation used above, we have

$$
T_{n}^{*}=n^{-1} \sum_{i=0}^{n-1} T^{* i}
$$

THEOREM 1. If

$$
\psi=\lim _{\nu} T_{n_{\nu}^{*}}^{*} \phi_{\nu}
$$

where each $\phi_{\nu}$ is a positive linear functional of norm 1 (i.e., $\phi_{\nu}$ satisfies conditions (1) and (3)) and $n_{\nu} \rightarrow \infty$, then $\psi \in L$.

Proof. It is clear that $\psi$ satisfies conditions (1) and (3). For any $x \in M$,

II am grateful to the referee for many suggestions for the improvement of an earlier version of this paper. Most important, by far, was his discovery of an error in what purported to be a proof that $Q=A$. 


$$
\begin{aligned}
\psi(x-T x) & =\lim _{\nu}\left[T_{n_{\nu}}{ }^{*} \phi_{\nu}(x)-T_{n_{\nu}}{ }^{*} \phi_{\nu}(T x)\right] \\
& =\lim _{\nu} \phi_{\nu}\left[n_{\nu}{ }^{-1} \sum_{i=0}^{n_{\nu}-1}\left(T^{i} x-T^{i+1} x\right)\right] \\
& =\lim _{\nu} \phi_{\nu}\left[n_{\nu}{ }^{-1}\left(x-T^{n}{ }_{\nu} x\right)\right] .
\end{aligned}
$$

But

$$
\left|\phi_{\nu}\left[n_{\nu}^{-1}\left(x-T^{n_{\nu}} x\right)\right]\right| \leqslant 2|| \phi_{\nu}|| \cdot n_{\nu}^{-1} \cdot\|x\| \rightarrow 0
$$

with $\nu$. Therefore, $\psi(x-T x)=0$.

The existence of generalized limits is an immediate consequence of Theorem 1 ; this is substantially the same proof that was described in the Introduction. Take any $\theta \in N$. Since $\left\{T_{n}{ }^{*} \theta\right\} \subset B^{*}$ and the latter set is compact, the former set must have limit points all of which are in $L$, according to Theorem 1 . We observe that $\left\{T_{n}{ }^{*} \theta\right\}$ is not a convergent sequence in $M^{*}$ for any $\theta \in N$, because convergence of $\left\{T_{n}{ }^{*} \theta\right\}$ in $M^{*}$ implies convergence of the sequence of numbers $\left\{T_{n}{ }^{*} \theta(x)\right\}$ for every $x \in M$. But, if $\theta=\theta_{p}$, then

$$
T_{n}^{*} \theta_{p}(x)=\theta_{p}\left(T_{n} x\right)
$$

is the $n$th arithmetic mean of the sequence $T^{p-1} x$, and it is easy to find an $x$ which will make $\left\{\theta_{p}\left(T_{n} x\right)\right\}$ diverge. What is not so obvious is that if $\omega \in \Omega$, $\left\{T_{n}{ }^{*} \omega\right\}$ need not converge either. Here is an example.

First, construct a sequence $\left\{\eta_{i}\right\}$ whose arithmetic means do not converge. Thus: $\eta_{1}=1, \eta_{i}=0$ if $2^{k} \leqslant i<2^{k}+2^{k-1}$, and $\eta_{i}=1$ if

$$
2^{k}+2^{k-1} \leqslant i<2^{k+1}, \quad k=1,2, \ldots
$$

Next, let

$$
\left\{\theta_{n_{k}}\right\}(k=1,2, \ldots)
$$

be a sequence in $N$ such that $n_{k}-n_{k-1} \geqslant 2^{k}$, and let $\omega$ be one of its limit points. Define $\xi_{n}=0$ if $n=n_{k}$ for some $k$ or $n<n_{1}$, and $\xi_{n}=\eta_{i}$ if $i$ is the least positive integer such that $n=n_{k}+i$ for some $k$. The inequality serves to guarantee that, for each $i$,

$$
\xi_{n_{k}+i}=\eta_{i}
$$

for all but a finite number of $k$ 's. Setting $x=\left\{\xi_{n}\right\}$, we have

$$
\omega(x)=\lim _{k \rightarrow \infty} \xi_{n_{k}}
$$

provided the limit exists, and more generally,

$$
\omega\left(T^{i} x\right)=\lim _{k \rightarrow \infty} \xi_{n_{k}+i} .
$$

It follows that for each $i, \omega\left(T^{i} x\right)=\eta_{i}$, and therefore,

$$
\left\{n^{-1} \sum_{i=0}^{n-1} \omega\left(T^{i} x\right)\right\}
$$

does not converge. But this implies that $\left\{T_{n}{ }^{*} \omega\right\}$ does not converge in $M^{*}$. 
The sequence $\left\{n_{k}\right\}$ is an example of a sequence of integers of density 0 in the number theoretic sense. Making use of the obvious one to one correspondence between $N$ and the set of positive integers, we may speak, in the same way, of non-dense subsets of $N$. It is somewhat surprising, in view of the fact that $N$ is a countable, dense, discrete set in $\Omega^{\prime}$, that there exist points $\omega$ in $\Omega$ which are not limit points of any non-dense subset of $N$. I conjecture that for some such $\omega$ the sequence $\left\{T_{n}{ }^{*} \omega\right\}$ does converge, but I have not been able to prove this. It will be seen in the proof of Theorem 3 that for each $x \in M$ there exists $\omega \in \Omega$ with the property that $\left\{T_{n}{ }^{*} \omega(x)\right\}$ is a convergent sequence of numbers.

3. Sets that generate $L$. We have already observed that $L \subset \mathfrak{S}(\Omega)$. Since $T_{n}{ }^{*}$ is a continuous mapping and $\Omega$ is compact, the set $A_{n}=T_{n}{ }^{*}(\Omega)$ is compact. From the additional fact that $T_{n}{ }^{*}$ is linear, it follows that for any set $S \subset B^{*}$,

$$
T_{n}{ }^{*}(\mathfrak{S}(S))=\mathfrak{S}\left(T_{n}^{*}(S)\right) .
$$

Since $L$ is elementwise invariant under $T_{n}{ }^{*}$, we have

$$
L=T_{n}{ }^{*}(L) \subset T_{n}{ }^{*}(\mathfrak{S}(\Omega))=\mathfrak{S}\left(T_{n}^{*}(\Omega)\right)=\mathfrak{S}\left(A_{n}\right) .
$$

It follows that

$$
L \subset \bigcap_{1}^{\infty} \mathfrak{S}\left(A_{n}\right)
$$

This conclusion is obtained so easily because the closed convex hull of a set is, in general, very much bigger than the set itself. We would like to have $L$ as the closed convex hull of an intersection rather than the intersection of hulls. It is hopeless, however, to expect that

$$
L=\mathfrak{S}\left(\bigcap_{1}^{\infty} A_{n}\right),
$$

because $\cap A_{n}$ is empty in the worst possible way; namely, the sets $A_{n}$ are mutually disjoint. To prove the last statement, fix $n$, and let $x=\left\{\xi_{i}\right\}$ where $\xi_{i}=1$ if $i$ is a multiple of $n$ and $\xi_{i}=0$ otherwise. Then for every $\omega \in \Omega^{\prime}$, $\omega(x)$ is either 0 or 1 , there is some integer $j^{\prime}$ such that $T^{* j^{\prime}} \omega(x)=1$, and $T^{* j} \omega(x)=1$ if $j \equiv j^{\prime}(\bmod n)$ and $T^{* j} \omega(x)=0$ if $j \not j^{\prime}(\bmod n)$. Consequently, $\phi(x)=1 / n$ for all $\phi \in A_{n}$, whereas, if $\phi \in A_{k}$ with $k<n$, then $\phi(x)=0$ or $1 / k$. Thus, $A_{k} \cap A_{n}$ is empty for all $k<n$ and for all $n$.

The operation on the sequence of sets $\left\{A_{n}\right\}$ that does the job we want is the topological limit superior. By definition,

$$
\psi \in \lim \sup A_{n}
$$

if every neighborhood of $\psi$ meets infinitely many of the sets $A_{n}$. Equivalently,

$$
\lim \sup A_{n}=\bigcap_{m=1}^{\infty} F_{m} \text {, }
$$


where $F_{m}$ is the closure of the set

$$
\bigcup_{n=m}^{\infty} A_{n}
$$

It is clear from the form of the sets $A_{n}$ that if $\psi \in \lim \sup A_{n}$, then there are directed systems

$$
\left\{T_{n_{\nu}}{ }^{*}\right\} \text { and }\left\{\omega_{\nu}\right\}
$$

directed by the same set $\{\nu\}$ and with $\omega_{\nu} \in \Omega$, such that $n_{\nu} \rightarrow \infty$ and

$$
T_{n_{\nu}}{ }^{*} \omega_{\nu} \rightarrow \psi
$$

Conversely, every cluster point of such a directed system is in $\lim \sup A_{n}$. Thus, $\lim \sup A_{n}$ is precisely the set $A$ of the introduction. By Theorem 1 , $A \subset L$, and since $L$ is compact and convex, we conclude that $\mathfrak{S}(A) \subset L$.

THEOREM 2. $\mathfrak{S}(A)=L$.

Proof. In view of the preceding discussion, we have only to prove that $L \subset \mathfrak{S}(A)$. But this follows easily from Theorem 2 of (4), which asserts that for a sequence $\left\{\mathfrak{S}\left(A_{n}\right)\right\}$ of compact convex sets,

$$
\lim \sup \mathfrak{S}\left(A_{n}\right) \subset \mathfrak{S}\left(\lim \sup A_{n}\right) .
$$

We have already seen that

$$
L \subset \bigcap_{1}^{\infty} \mathfrak{S}\left(A_{n}\right)
$$

which, in turn, is contained in $\lim \sup \mathfrak{S}\left(A_{n}\right)$.

Corollary 1. All of the extreme points of L are in $A$.

Proof. The topological limit superior is always a closed set. According to a theorem of Milman (9; 3, p. 84, prop. 4), all of the extreme points of the closed convex hull of a set are in the closure of that set.

Corollary 2.

$$
L=\bigcap_{n=1}^{\infty} T_{n} *(\mathfrak{S}(\Omega)) .
$$

This can be proved directly, but at this stage it comes very easily out of a string of inequalities:

$$
\begin{aligned}
L= & \bigcap_{n=1}^{\infty} T_{n}{ }^{*}(L) \subset \cap T_{n}{ }^{*}(\mathfrak{S}(\Omega))=\cap \mathfrak{S}\left(A_{n}\right) \\
& \subset \lim \sup \mathfrak{S}\left(A_{n}\right) \subset \mathfrak{S}\left(\lim \sup A_{n}\right)=L .
\end{aligned}
$$

We turn now to the development of a sharper result. It is well known that $M$ is isomorphic and isometric (equivalent in Banach's sense) with the space of all continuous functions on the compact set $\Omega^{\prime}$, the isomorphism taking the element $x \in M$ into the function whose value at $\omega \in \Omega^{\prime}$ is $\omega(x)$. It is convenient 
to identify the two spaces and use the notation $x(\omega)$ to denote $\omega(x)$. To each continuous linear functional $\phi$ on $M$, there corresponds (5) a unique regular Borel measure $m$ on $\Omega^{\prime}$ with the property

$$
\phi(x)=\int_{\Omega^{\prime}} x(\omega) d m(\omega) \text { for all } x \in M .
$$

Conditions $(1)-(4)$ on the functional $\phi$ may be translated into the following conditions on the measure $m$ : For every Borel set $\Gamma$,

$\left(1^{\prime}\right) m(\Gamma) \geqslant 0$

$\left(2^{\prime}\right) m\left(T^{*} \Gamma\right)=m(\Gamma)$;

$\left(3^{\prime}\right) m\left(\Omega^{\prime}\right)=1$;

(4') $m(N)=0$.

Theorem 3. If $Q$ is the set of all limit points of sequences $\left\{T_{n}{ }^{*} \omega\right\}, \omega \in \Omega$, then $\mathfrak{S}(Q)=L$.

Proof. We apply here the technique of Milman, as described in (4). Since $L$ is a compact, convex set and $Q \subset L$, it suffices to prove that for every continuous (in the weak* topology) linear functional $f$ on $M^{*}$,

$$
\sup _{\psi \in L} f(\psi)=\sup _{\psi \in Q} f(\psi) .
$$

But every continuous linear functional on $M^{*}$ comes from an element of $M$; that is, given such an $f$, there exists $x \in M$ such that $f(\phi)=\phi(x)$ for all $\phi \in M^{*}$. We wish to prove, then, that for all $x \in M$,

$$
\sup _{\psi \in L} \psi(x)=\sup _{\psi \in Q} \psi(x) .
$$

Fix $x \in M$. Since $L$ is compact, there is an element $\psi_{0}$ in $L$ such that

$$
\psi_{0}(x)=\sup _{\psi \in L} \psi(x) .
$$

Let $m_{0}$ be the regular Borel measure on $\Omega^{\prime}$ corresponding to the functional $\psi_{0}$. Conditions $\left(1^{\prime}\right)-\left(4^{\prime}\right)$ are satisfied by $m_{0}$, so that all of the measure is carried by the set $\Omega: m_{0}(\Omega)=1$. The individual ergodic theorem (6) is applicable to this situation, and we conclude that $\lim _{n \rightarrow \infty} T_{n} x(\omega)$ exists for all $\omega$ in an invariant (under $T^{*}$ ) subset $\Delta$ of $\Omega$ of $m_{0}$-measure 1 . If we let $X(\omega)$ denote this limit for $\omega \in \Delta$, and $X(\omega)=0$ for $\omega \in \Omega-\Delta$, then the ergodic theorem states further that $X$ is a measurable function, invariant under $T^{*}$, and

$$
\int_{\Omega} X(\omega) d m_{0}(\omega)=\int_{\Omega} x(\omega) d m_{0}=\psi_{0}(x) .
$$

For each $\omega \in \Delta$, the set $\left\{T_{n}{ }^{*} \omega\right\}$ has limit points, and according to Theorem 1 , every such limit point is in $L$. Consequently, there is a $\psi$ in $L$, which depends upon $\omega$ and such that

$$
\lim _{n \rightarrow \infty} T_{n} x(\omega)=\psi(x) .
$$


Since $\psi(x) \leqslant \psi_{0}(x)$ for all $\psi \in L$, we have

$$
X(\omega)=\lim T_{n} x(\omega) \leqslant \psi_{0}(x)
$$

for all $\omega \in \Delta$. This inequality together with equation (8) and the fact that $m_{0}(\Delta)=1$ implies that $X(\omega)=\psi_{0}(x)$ for almost all $\omega \in \Delta$. In particular, there is at least one $\omega_{0}$ for which the equality holds, and any limit point $\chi$ of $\left\{T_{n}{ }^{*} \omega_{0}\right\}$ has the properties:

$$
\chi \in Q \text { and } \chi(x)=\psi_{0}(x)=\sup _{\psi \in L} \psi(x) .
$$

This verifies (7) and completes the proof of the theorem (ci. $6 \mathbf{a}$ ).

4. Almost convergent sequences. Lorentz (7) calls a sequence $x \in M$ almost convergent if $\psi(x)$ is independent of $\psi \in L$. By Theorem 3 , it is obviously: sufficient to require that $\psi(x)$ be constant for $\psi \in Q$. Observing that every $\psi \in Q$ has the form

$$
\psi(x)=\lim _{\nu} T_{n_{\nu}} x(\omega), \omega \in \Omega,
$$

leads to the following characterization of almost convergent sequences:

LEMmA. In order that there exist a number $\sigma$ such that $\psi(x)=\sigma$ for all $\psi \in L$, it is necessary and sufficient that

$$
\lim _{n \rightarrow \infty} T_{n} x(\omega)=\sigma
$$

for all $\omega \in \Omega^{\prime}$.

(We write $\Omega^{\prime}$ rather than $\Omega$ in order to facilitate the coming discussion. Both are correct.)

The known characterization of almost convergent sequences is the following (7; see also $\mathbf{1 0}$, p. 53):

THEOREM 4. A necessary and sufficient condition for the existence of $\sigma$ such that $\psi(x)=\sigma$ for all $\psi \in L$ is that

$$
\lim _{n \rightarrow \infty} \frac{1}{n} \sum_{i=1}^{n} \xi_{i+k}=\sigma,
$$

uniformly in $k$.

In our notation, (10) takes the form

$$
\lim _{n \rightarrow \infty} T_{n} x(k)=\sigma,
$$

uniformly in $k(\in N)$. Since $N$ is dense in $\Omega^{\prime}$ and $T_{n} x$ is a continuous function on $\Omega^{\prime},\left(10^{\prime}\right)$ obviously implies $(9)$. But the converse is also true. For (9) implies weak convergence of the sequence $\left\{T_{n} x\right\}$ in $M$ to the constant $\sigma$. By the mean ergodic theorem in Banach space (6), this implies convergence in norm, i.e. uniform convergence on all of $\Omega^{\prime}$. 
5. The maximal generalized limit of a given sequence. If $\rho$ is a functional on $M$ satisfying the two conditions

(i) $\rho(x+y) \leqslant \rho(x)+\rho(y)$,

(ii) $\rho(\lambda x)=\lambda \rho(x)$ for all $x, y \in M$ and $\lambda \geqslant 0$,

then, according to the Hahn-Banach theorem, there exists a linear functional $\phi$ such that $\phi(x) \leqslant \rho(x)$ for all $x$. The functional $\tau$ defined by

$$
\tau(x)=\sup _{\psi \in L} \psi(x)
$$

satisfies conditions (i) and (ii). Noting that for a linear functional $\phi, \phi(x) \leqslant \tau(x)$ for all $x \in M$ implies

$$
-\tau(-x) \leqslant-\phi(-x)=\phi(x) \leqslant \tau(x),
$$

one sees that every such $\phi$ is actually a generalized limit.

It is clear from the proof of the Hahn-Banach theorem (2, p. 28) that not only is there a linear functional $\phi$ dominated by $\rho$, but also, if $x_{0} \in M$ is given, $\phi$ may be chosen so that $\phi\left(x_{0}\right)=\rho\left(x_{0}\right)$. Consequently, if $\rho$ has the property that $\phi \leqslant \rho$ implies $\phi \in L$, then $\rho(x) \leqslant \tau(x)$ for all $x \in M$. In Banach's proof of the existence of generalized limits the functional which is used for $\rho$ is

$$
\tau^{\prime}(x)=\inf \limsup _{k \rightarrow \infty} n^{-1} \sum_{i=1}^{n} \xi_{k+m i},
$$

where the infimum is taken over all possible choices of non-negative integers $m_{1}, \ldots, m_{n}$. By what was said earlier, $\tau^{\prime}(x) \leqslant \tau(x)$, and it is easy to reverse this inequality to obtain $\tau^{\prime}(x)=\tau(x)$. All of this has been observed before (7).

Now, let us use Theorem 2 to calculate $\tau(x)$ from the terms of the sequence $\left\{\xi_{n}\right\}$. Since $L=\mathfrak{S}(A)$, where $A=\lim \sup A_{n}$, we have

$$
\tau(x)=\sup _{\psi \in A} \psi(x) .
$$

By definition,

$$
A=\bigcap_{m=1}^{\infty} F_{m}
$$

where $F_{m}$ is the closure of

$$
\bigcup_{n=m}^{\infty} A_{n}
$$

It is easy to see that

$$
\sup _{\phi \in A n} \phi(x)=\limsup _{k \rightarrow \infty} n^{-1} \sum_{i=0}^{n-1} \xi_{k+i}
$$


so that

$$
\sup _{\phi \in F_{m}} \phi(x)=\sup _{n \geqslant m} \limsup _{k \rightarrow \infty} n^{-1} \sum_{i=0}^{n-1} \xi_{k+i}
$$

and finally,

$$
\sup _{\psi \in A} \psi(x) \leqslant \inf _{m} \sup _{n \geqslant m} \limsup _{k \rightarrow \infty} n^{-1} \sum_{i=0}^{n-1} \xi_{k+i} .
$$

We denote the right side of the inequality by $\tau^{\prime \prime}(x)$ :

$$
\tau^{\prime \prime}(x)=\limsup _{n \rightarrow \infty} \limsup _{k \rightarrow \infty} n^{-1} \sum_{i=0}^{n-1} \xi_{k+i} .
$$

Since $F_{m}$ is a compact set, the supremum of $\phi(x)$ is attained at some $\phi_{m} \in F_{m}$, for each $m$. The sequence $\left\{\phi_{m}\right\}$ has limit points and every one of these limit points is in $A$. It follows that the inequality in (11) is actually an equality.

Theorem 5. For every $x=\left\{\xi_{n}\right\}$ in $M$,

$$
\sup _{\psi \in L} \psi(x)=\tau^{\prime}(x)=\tau^{\prime \prime}(x)=\lim _{n \rightarrow \infty} \limsup _{k \rightarrow \infty} n^{-1} \sum_{i=0}^{n-1} \xi_{k+i} .
$$

Everything has already been proved excepting the existence of the last limit, which is obtained by observing that

$$
\tau^{\prime}(x) \leqslant \liminf _{n \rightarrow \infty} \limsup _{k \rightarrow \infty} n^{-1} \sum_{i=0}^{n-1} \xi_{k+i} \leqslant \tau^{\prime \prime}(x) .
$$

An alternate proof of the equality $\tau^{\prime \prime}=\tau$ can be given by using the HahnBanach argument mentioned earlier; namely, if $\phi$ is a linear functional such that $\phi(x) \leqslant \tau^{\prime \prime}(x)$ for all $x$, then $\phi \in L$. Consequently, $\tau^{\prime \prime} \leqslant \tau$, and a simple computation shows that $\psi \in L$ implies $\psi \leqslant \tau^{\prime \prime}$. The argument based upon Theorem 2, although more complicated than this one, has the advantage that it enables one to discover the form of the functional $\tau^{\prime \prime}$. In fact, the equality $\tau^{\prime}=\tau^{\prime \prime}$ does not seem to have been noticed before.

It ought to be possible to prove that $\tau^{\prime}=\tau^{\prime \prime}$ directly from the expressions for $\tau^{\prime}$ and $\tau^{\prime \prime}$ in terms of the sequence $\left\{\xi_{n}\right\}$, and this was done by Professor J. H. B. Kemperman. The essential step in his proof (unpublished) is the following inequality: if

$$
0=m_{1} \leqslant m_{2} \leqslant \ldots \leqslant m_{n},
$$

and $p$ is any positive integer, then

$$
\limsup _{k \rightarrow \infty} p^{-1} \sum_{i=1}^{n} \xi_{k+i} \leqslant \limsup _{k \rightarrow \infty} n^{-1} \sum_{i=1}^{n} \xi_{k+m i}+\frac{2\|x\|}{n p}(n-1)\left(m_{n}-m_{1}\right) .
$$




\section{ReferenCes}

1. R. F. Arens and J. L. Kelly, Characterizaiions of the space of continuous functions over a compact Hausdorff space, Trans. Amer. Math. Soc., 62 (1947), 499-508.

2. S. Banach, Théorie des opérations linéaires (Warsaw 1932).

3. N. Bourbaki, Espaces vectorieis topologiques (Actualités scientifiques et industrielles, no. 1189, Paris, 1953).

4. M. Jerison, A property of extreme points of compact convex sets, Proc. Amer. Math. Soc., 5 (1954), 782-783.

5. S. Kakutani, Concrete representations of abstract (M)-spaces, Aml. of Math., 42 (1941), 994-1024.

6. S. Kakutani, Ergodic theory, Proc. Internat. Congr. Math., Cambridge, 1950, II, 128-142.

6a. N. Kryloff and N. Bogoliouboff, La théorie générale de la mesure dans son application à l'étude des systèmes dynamiques de la mécanique non linéaire, Ann. of Math., 38 (1937), 65-113.

7. G. G. Lorentz, A contribution to the theory of divergent sequences, Acta Math., 80 (1948), 167-190.

8. S. Mazur, On the generalized limit of bounded sequences, Colloq. Math., 2 (1951), 173-175.

9. D. Milman, Characteristics of extremal points of regularly convex sets, Doklady Akad. Nauk SSSR (N.S.), 57 (1947), 119-122.

10. J. von Neumann, Invariant Measures (Institute for Advanced Study, Princeton, 19401941).

Purdue University 